\title{
Electrical Conduction in Triarylmethane Halides and Thiocyanates
}

\author{
K. Tennakone, M. Kahanda and C. Kasige \\ Department of Physics, Ruhuna University Coliege, Matara, Sri Lanku
}

(Date of receipt: 09 August 1983)

(Date of acceptance: 03 April 1984)

\begin{abstract}
The temperature variation of the conductivity of triarylmethane halides and thiocyanates are studied. It is found that in halides the thermal activation energies of charge carriers increase in the sequence $\mathrm{I}>\mathrm{Br}>\mathrm{Cl}$. Thiocynates show a conductivity transition at $\operatorname{cs} 126^{\circ} \mathrm{C}$.
\end{abstract}

\section{Introduction}

It is well known that organic charge transfer (CT) complexes in the solid state frequently exhibit semiconduction. $.^{3}, 4$ The mechanism of conduction in these molecular solids is believed to be the hopping of charge carriers ${ }^{2},{ }^{8}$ from donor to acceptor sites. Some of the simplest and most extensively investigated organic CT solids are the dyes. ${ }^{4},{ }^{6}$ To understand the nature of electrical transport in CT complexes, it is desirable to study the conductivity properties of cationic (anionic) dyes when the donor (acceptor) site is a simple anion (cation) of known properties. In this paper we report our measurements of the conductivity of triarylmethane (TR) dyes, when the anionic ligand is a halide or a pseudohalide.

\section{Experimental}

TR dyes (Metyl Violet and Rosaniline) commercially supplied as chlorides (BDH brand) are purified by recrystallization. To prepare the corresponding iodides an alcoholic solution of the dye and potassium iodide is boiled until most alcohol is expelled. The TR iodides insoluble in aqueous $\mathrm{KI}$, separated by filtration are recrystallized and dried in vacuum at $105^{\circ} \mathrm{C}$. The thiocyanates of TRs can be prepared by the same method, when KI is replaced by KCNS. However, the double decomposition does not give a good yield of the bromide. TR bromides are prepared by treating the correspoinding leuco base with $\mathrm{HBr}$. Purified samples free from moisture are pressed into pellets between carbon electrodes in a pyrex tube (diameter $\tilde{s} 0.4 \mathrm{~cm}$, pellet length $\tilde{\omega} 0.2 \mathrm{~cm}$ ) at a pressure of $10^{\circ} \mathrm{Pa}$. Ends of the tube are sealed with epoxy resin and the conductivity at different temperatures is determined using a d. c. resistance meter. Very thin discs of the material compacted in sealed tubes are used to pass large currents for long intervals of time to see whether ionic conduction is present. Conductivity characteristics remained linear at constant slope indicating that ionic conduction is absent.

Attempts to determine the mobilities by the measurement of Hall voltages were unsuccessful. 


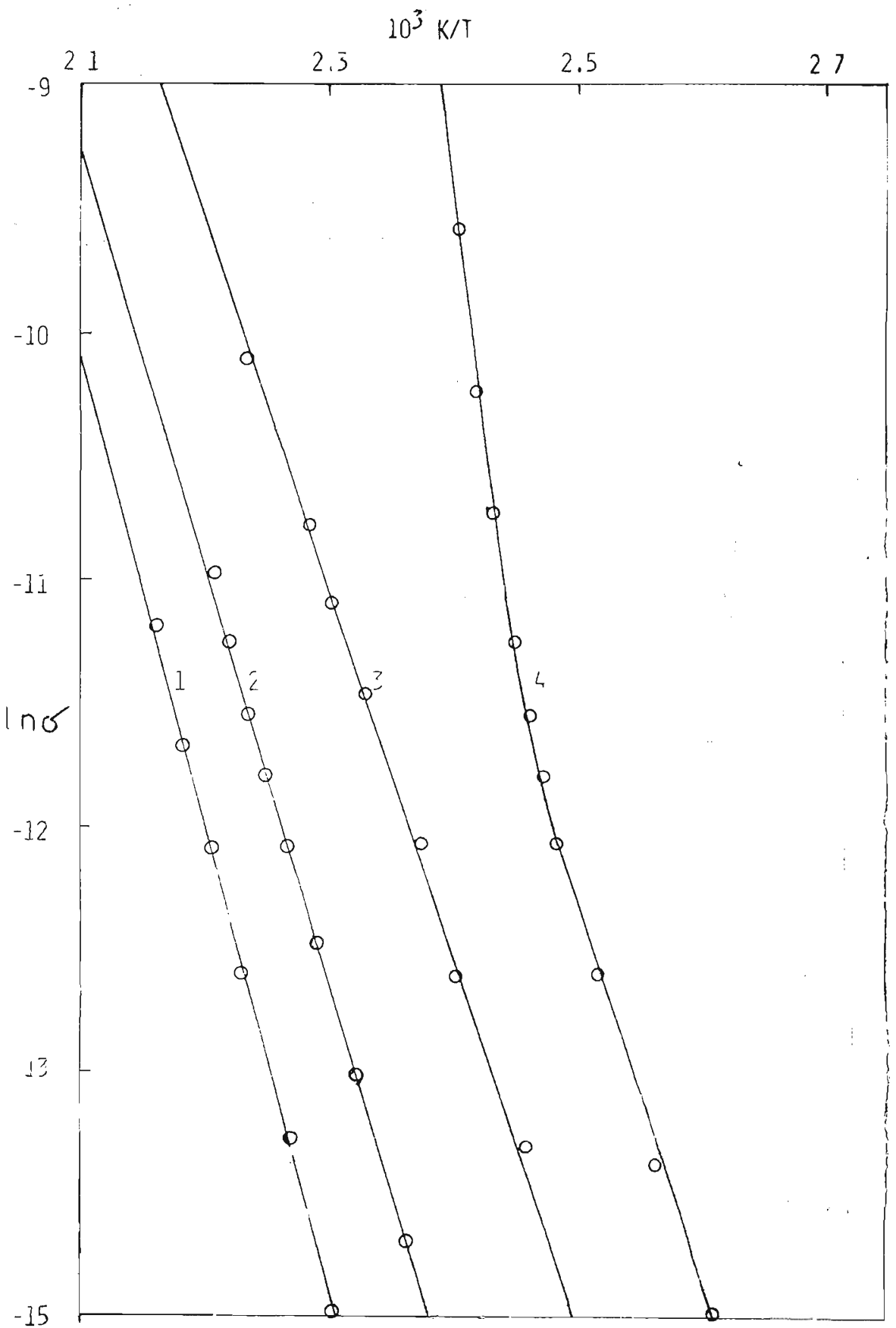

Figure 1. Plot of $\ln \sigma$ ( $\sigma$ in $\Omega-\mathrm{m}^{-1}$ ) for halides and thiocyanates of Metyl Violet. (1) Chloride (2) Bromide (3) Iodide (4) Thiocyanate. 


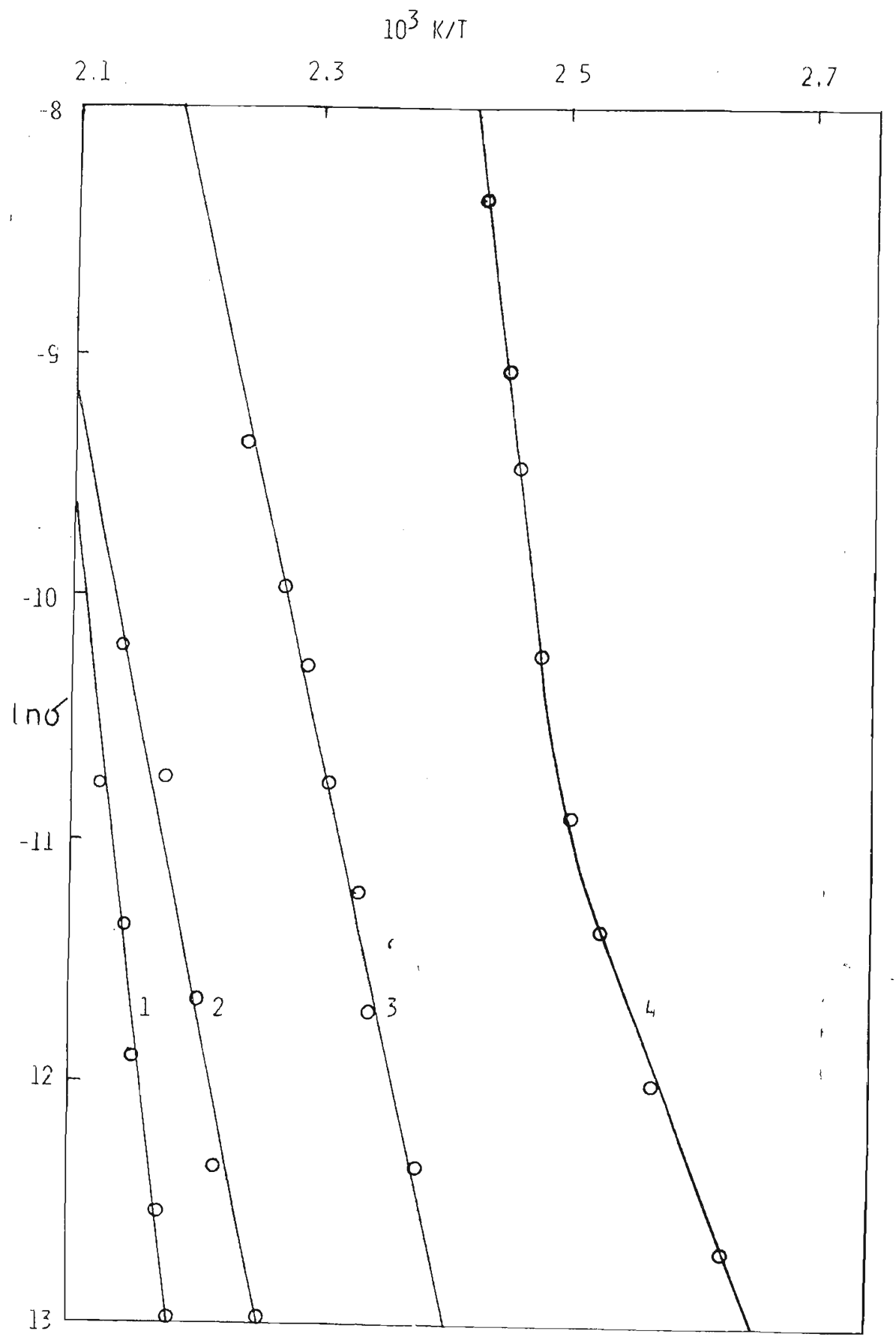

Figure 2. Plot of $\ln \sigma$ ( $\sigma$ in $\Omega^{-1} \mathrm{~m}^{-1}$ ) for halides and thiocyanates of Rosaniline. (1) Chloride (2) Bromide (3) Iodide (4) Thiocyanate. 


\section{Results and Discussion}

The plots of $l n v s T^{-1}$ for metyl violet and rosaniline are given in Figures (1) and (2). In the case of TR halides, $\sigma$ varies linearly with $T^{-1}$, throughout the entire temperature range, $\left(30^{\circ} \mathrm{C}-\right.$ M.P. $270,210^{\circ} \mathrm{C}$ for rosaniline and metyl violet respectively) indicating that the relation

$$
\sigma=\sigma_{\circ} e^{-E^{\prime} / k t}
$$

is satisfied. In thiocyanates the data fits into two straight line portions with a transition temperature $\tilde{y}_{2} 126^{\circ} \mathrm{C}$. The activations energies $(E)$ are given in Table 1 .

TABLE 1. Thermal activation energies of Metyl Violet and Rosaniline halides and thiocyanates

\begin{tabular}{lll} 
& \multicolumn{2}{c}{ E/eV } \\
Anion & Metyl Violet & Rosaniline \\
\hline Cl- & 1.6 & 3.6 \\
Br- & 1.4 & 2.3 \\
I- & $1.3\left(\mathrm{~T}<126^{\circ} \mathrm{C}\right)$ & 2.0 \\
CNS- & $1.5\left(\mathrm{~T}<126^{\circ} \mathrm{C}\right)$ \\
& $3.3\left(\mathrm{~T}>126^{\circ} \mathrm{C}\right)$ & $3.4\left(\mathrm{~T}>126^{\circ} \mathrm{C}\right)$ \\
\hline
\end{tabular}

It is secn that the values of $E$ show a progressive decrease in the order chloride, bromide, iodide. This could be attributed to decrease in ionization energies of the halide ions. The thermal activation energy associated with hopping conduction is generally taken as, ${ }^{5}$

$$
E=I-A-P
$$

where $I=$ ionization energy at the donor site, $A=$ electron affinity at the acceptor site, $P=$ polarization energy. The value of $A$ should be nearly same for all TR halides. Since therc is no reliable way of estimating $P$, a quantitative verification of (2) is not possible.

All thiocyanated $T R$ show a transition at $\mathrm{T}$ \& $126^{\circ} \mathrm{C}$, with an abrupt change in the activation energy. How this transition originates remains unresolved.

As the absolute purification of these compounds is very difficult, different samples give slightly different but similar results. The room temperature conductivity shows about $20-30 \%$ variation. However, the activation energies remain nearly constant.

\section{References}

1. Austrn, I. G. \& Motr, N. F. (1969). Adv. Philys. 18: 41.

2. Austin, I. G. \& Motr, N. F. (1970). Science, 168; 71.

3. BraUn, C. L. (1979). in Hand book on Semiconductors, Vol. 3 ed. S. P. Keller (NorthHolland, Amsterdam).

4. Gurmainn, F. \& Lyons, L. E. (1967). Organic semicondictors (John Wiley, New York).

5. Lyons, L. E. (1957). J. Chem. Soc. 5001.

6. MEIER, H. (1974). Organic semiconductors Dark and Photocontuctivity of orgamic solitis (Verlag chemie, Wienheim).

7. Motr, N. F: (1956). Canadian Journal of Phys. 34; 1356.

8. Spear, w. E. (1974). Adv. Phys. 23; 523. 